\title{
How Context and User Behavior Affect Indoor Navigation Assistance for Blind People
}

\author{
João Guerreiro' ${ }^{1}$, Eshed Ohn-Bar ${ }^{1}$, Dragan Ahmetovic ${ }^{1}$, Kris Kitani $^{1}$, Chieko Asakawa ${ }^{1,2}$ \\ ${ }^{1}$ Carnegie Mellon University, ${ }^{2}$ BBM Research - Tokyo \\ \{jpvguerreiro, eshed1, dragan1\}@cmu.edu, \{kkitani, chiekoa\}@cs.cmu.edu
}

\begin{abstract}
Recent techniques for indoor localization are now able to support practical, accurate turn-by-turn navigation for people with visual impairments (PVI). Understanding user behavior as it relates to situational contexts can be used to improve the ability of the interface to adapt to problematic scenarios, and consequently reduce navigation errors. This work performs a fine-grained analysis of user behavior during indoor assisted navigation, outlining different scenarios where user behavior (either with a white-cane or a guide-dog) is likely to cause navigation errors. The scenarios include certain instructions (e.g., slight turns, approaching turns), cases of error recovery, and the surrounding environment (e.g., open spaces and landmarks). We discuss the findings and lessons learned from a real-world user study to guide future directions for the development of assistive navigation interfaces that consider the users' behavior and coping mechanisms.
\end{abstract}

\section{CCS Concepts}

•Human-centered computing $\rightarrow$ Empirical studies in accessibility; Accessibility technologies; User studies; Field studies;

\section{Keywords}

Blind navigation; people with visual impairments; navigation strategies; assistive technologies, user behavior.

\section{INTRODUCTION}

People with Visual Impairments (PVI) often need the assistance of sighted guides when visiting unfamiliar or complex indoor environments [18]. Current research in assistive navigation technologies aims to increase the independence of PVI by supporting and complementing their Orientation and Mobility (O\&M) skills. For instance, interactive accessible maps [4] and virtual navigation [8] help building a mental representation of the environment before visiting it;

Permission to make digital or hard copies of all or part of this work for personal or classroom use is granted without fee provided that copies are not made or distributed for profit or commercial advantage and that copies bear this notice and the full citation on the first page. Copyrights for components of this work owned by others than ACM must be honored. Abstracting with credit is permitted. To copy otherwise, or republish, to post on servers or to redistribute to lists, requires prior specific permission and/or a fee. Request permissions from permissions@acm.org.

W4A '18, April 23-25, 2018, Lyon, France

(C) 2018 ACM. ISBN 978-1-4503-5651-0/18/04 . \$ $\$ 15.00$

DOI: https://doi.org/10.1145/3192714.3192829 computer vision approaches enable object recognition and obstacle avoidance without physical contact [7, 11, 19]; and indoor positioning techniques provide accurate localization and guidance using turn-by-turn navigation [2, 5, 15].

The combination of increased localization accuracy [2] and interfaces that comply with O\&M training [17], new standards for navigation assistance (more details in [14]) and knowledge about the behaviors, preferences and coping mechanisms of PVI during navigation (e.g., $[1,12,18]$ ) has resulted in effective navigation systems with a low number of errors [15]. While such errors may be caused by an occasional decrease in localization accuracy, we noticed that they can also be caused by problematic situations combined with specific/unexpected user behavior or their coping mechanisms when interacting with the system.

In related research, navigation performance is often analyzed in terms of error rates and route completion times. Alternatively, self-reported feedback is evaluated based on surveys and interviews. Our study complements these works by providing an in-depth analysis of PVI performing navigation tasks along the route. Such approach is essential for understanding causes of navigation errors, in particular in cases where the system behaves as expected. By observing PVI in two user studies with an accurate navigation system [15], we present a thematic analysis which outlines specific situations and user behaviors that are likely to cause navigation errors. We present lessons learned that can be leveraged by navigation interfaces in order to adapt and cope with these problematic scenarios and observed behaviors.

\section{METHOD}

Our main goal is to understand the causes for navigation errors when using an accurate navigation system for PVI. This knowledge can benefit future developments in assisted navigation by providing insights that consider the users' behavior and their coping mechanisms with the system.

\subsection{Apparatus}

NavCog [15] is a smartphone-based indoor navigation system for PVI that provides turn-by-turn guidance and information about relevant landmarks and POIs within close proximity to the user. At the start of each segment, it reads the next instruction (e.g., "proceed 70 feet and turn left"). As the user proceeds, NavCog provides periodic information about the remaining distance and an "approaching" message right before the turn. At the turning point, the system provides a verbal instruction (e.g., "turn left") and a short vibration and sound effect. When the user completes the 
turn, achieving the correct orientation, the vibration and sound are provided again.

NavCog localization engine combines BLE beacon fingerprinting and a pedestrian motion model (more details in $[2,15])$, achieving an average localization error under 1.65 meters in previous studies [2, 15]. We instrumented three buildings at the Carnegie Mellon University (CMU) with the NavCog environment, covering an area of $58,800 \mathrm{~m}^{2}$.

\subsection{User Studies}

We observe videos of navigation tasks during two broader user studies with PVI using NavCog at the CMU campus. In the first study, 13 blind participants (six guide-dog and seven white-cane users - light perception at most) performed a set of tasks with NavCog. For this observation, we only considered navigation tasks where the goal was to follow the instructions and reach the destination. This study comprised both short (a single floor, 200 feet, four turns and six POIs/landmarks) and long (two floors, using an elevator, 690 feet, eleven turns and 22 POIs/landmarks) routes.

In the second study (more details in [13]), 8 blind (whitecane) participants repeated a set of navigation tasks (in two different routes) using NavCog. The two routes had 500 and 250 feet length, 8 and 7 turns, and 13 and 10 POIs and landmarks, respectively. In both studies, video was recorded for analysis purposes from a first- (participant) and thirdperson (researcher) perspective, using two GoPRO cameras. Participants were compensated for their time ( $\$ 25$ per hour).

\section{FINDINGS}

Based on our observations, we identified a set of situational contexts where certain user behaviors caused navigation errors. The errors may be caused either by users' navigation strategies or coping mechanisms as they interact further with the system. In this section, we summarize the main findings of our thematic analysis and outline a set of lessons learned that can guide the development of future navigation assistance interfaces.

\subsection{Close Sequence of Instructions}

The navigation instructions are announced by NavCog at the time and location that the user needs to act. With high localization accuracy this approach resulted in very few navigation errors for all participants. In general, after performing a turn, users start walking while listening to the next instruction. This is only a problem when there is a very close sequence of actions/instructions, such as turn left, proceed 10 feet, turn right (Figure 1). In this case, faster users (often guide-dog users) that immediately start walking may perform a late second turn. Another example is the one of landmarks (e.g., a door) that are located right after a turn, which may influence the user's perception of the environment. As a door was only announced after performing the turn, some users thought they were at a wrong location because they did not feel any open path on the respective side. This is supported by users' feedback, who suggested using a single instruction in close consecutive turns (turn right, and after 10 feet turn left) and landmarks (turn right and find a door or go through a door and turn right).

A close sequence of instructions should be combined into a single one. This can increase contextual awareness and prepare the user for the fast sequence of steps.

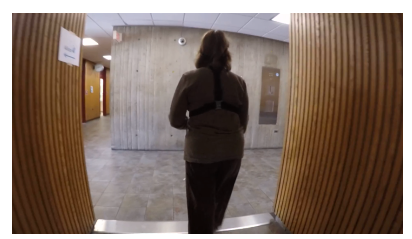

(a)

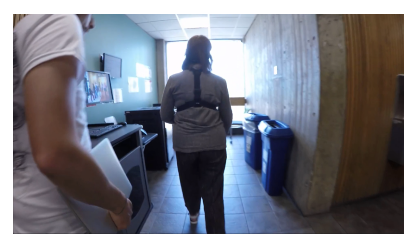

(b)
Figure 1: An example of two consecutive turns: After completing the first turn (a), separate instructions can cause a late turn (b). Users can leverage the knowledge of the next action, particularly in cases of short spatial and temporal proximity.

\subsection{Approaching a Turn}

During both user studies most participants highlighted the positive impact of the approaching instruction, since being prepared allowed them to rarely miss a turn. While some participants kept their pace, others noticeably reduced their speed to make sure they turn at the right location. Another common behavior was either to prompt their dog to turn or to sense the wall with the white-cane in order to find an open path. This is very likely a coping mechanism due to a previous late turn caused by an higher localization error (e.g., two or three meters can cause the user to perform a late turn). Such behavior was still successful in most occasions, except when there was a very close sequence of intersections so that an early turn resulted in following an alternative path (instead of sensing the wall). Such sequence of intersections is also more likely to result in a navigation error when there is a decrease in localization accuracy.

The approaching instruction is valuable, but additional mechanisms are needed when there are close alternative turns. In those cases, alternative ways to communicate the approaching or additional context needs to be provided. Some examples include delaying the approaching instruction or informing about the sequence of intersections to prevent an early turn.

When arriving to a decision point and not prompted to make a turn, guide dogs often reduce their speed or stop. This often overlaps with a successful turn instruction and consequent action. Occasionally, guide dogs stop slightly before NavCog announces the turn. This may be due to a minor localization error (e.g., 1 meter) or because the corridor is wide and the dog stops at the beginning of the intersection. In these cases, although there is an open path, participants needed to take one or two additional steps in order for the system to announce the turn. An alternative behavior was for the dog to take the lead, for instance by following a known route (e.g., the path taken - in the opposite direction - to the route starting point) or other pedestrians. When that resulted in an error and involved a clear change in direction, users were quickly able to understand and correct their dog. However, it was more difficult to perceive slight changes in direction. In these cases, either the user perceives that a mistake has been made or the system alerts and corrects the user after a certain threshold.

Recognizing an error takes longer in unnoticed slight turns. In particular with guide-dog users, systems should be prepared to identify potential areas for difficult error recognition and act sooner to correct the user or, when there is uncertainty, alert the user that the system is not confident about his/her location. 




(a)

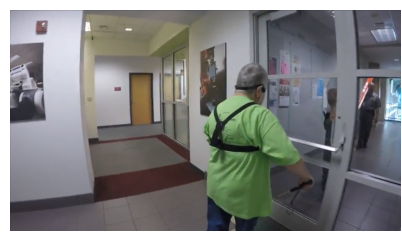

(b)
Figure 2: Two example scenarios from the user study which motivate our thematic analysis. (a) The user is directed to go forward, but veering in the open space results in confusion near the chair area on the right. (b) The user is directed to make a slight right turn in order to align with the new position of the corridor, yet an over-turn leads her to a door that she thought to be part of the path.

\subsection{Error Recovery}

Guide dog users rarely need to recover from errors as the dog follows the correct path when instructed a little earlier or at the turn location. When instructed late (for instance due to their faster pace), the dog often looks for the next turn, which results in longer recovery times. Still, users with good echolocation skills [10] were able to recover quickly by perceiving that they had just passed an intersection (which also occurred for white-cane users). One participant commented "So I was using (..) my echolocation skills to figure out.. OK I just passed a corridor, it must have meant to go there". In general, white-cane users also made a low number of navigation errors. In most cases, they recovered very quickly as they could find the correct path just by moving their cane backward and forward to find an opening. Longer recovery times occur in wider corridors or open areas due to the absence of a close wall. In addition, an occasional increase in localization error may result in the user moving further from the turning point. In these cases, it can result in the user going back and forth to find an open corridor.

The system should detect unexpected and erroneous behavior and intervene to help the user recover. While off-route and wrong-direction identification is valuable to recover from errors and re-direct the user, it uses a distance threshold. Identifying erroneous behavior (e.g., going back and forth) before reaching that threshold may result in sooner intervention and recovery.

\subsection{Slight Turns}

Instructions to turn right or left often resulted in approximately 90 degree turns, which are prevalent in both indoor and outdoor environments [3]. However, when instructed to make a slight turn (between 30 and 60 degrees), white-cane users often over-turned (Figure 2 (b)). Although NavCog provides a short vibration and sound effect when reaching the correct orientation, users' end up receiving the feedback after finalizing the turn, which is often performed with a single, continuous movement. In general, users were able to quickly recover after sensing the wall, but referred to the ambiguity of slight-turns as the main cause for over-turning. The literature refers to different alternatives to provide directional information, but there is no strong preference and evidence towards a particular one. For instance, cardinal directions may be difficult to identify [16], in particular in indoor locations. On the other hand, degrees or clockwise information enable higher granularity instructions, but sim- plified instructions are often preferred by users [9].

Guide-dog users were equally able to perform regular and slight turns, as they always provided right/left instructions to the dog. Their feedback suggests that very slight turns are not required to be mentioned, unless there are multiple alternatives and a need to disambiguate the correct path.

Additional feedback should be provided in case of over-turning. Besides providing feedback about reaching a certain angle (success), it is necessary to correct the users when their orientation is not the expected (by informing that the user over-turned through speech or sonification). In addition, further investigation needs to be conducted on how to convey directional instructions in a way that minimizes errors, in particular in slight turns.

\subsection{Corridors and Open Spaces}

Due to their O\&M skills, white-cane users often navigated throughout the corridors without sensing the walls with their cane. Exceptions occur when approaching a turn (as previously mentioned) and sometimes as a confirmation when instructed to turn at the end of the corridor. In addition, it may occur due to veering (a change in direction), which is quickly corrected in narrow corridors as the cane hits the wall, but is more challenging in wide corridors and open areas (Figure $2(\mathrm{a})$ ) as it may cause the user to deviate from the intended path or bump into obstacles $[18,6]$. While NavCog has mechanisms to deal with veering (by correcting the user to "bear right/left"), it needs to be somewhat conservative to avoid overwhelming the user when the veering is minor (or nonexistent due to orientation error).

Increase the accuracy in open-areas in order to quickly detect and correct veering. Designers should concentrate their efforts to increase localization (and orientation) accuracy in situations where the user has more chances to deviate from the path. Better localization can be accomplished in several ways depending on the technology used (e.g., by placing additional beacons). Yet, in practice it is likely that current tools will continue to present localization and orientation errors. In such cases, the system could inform the user of its uncertainty so that users may cope with the situation in a more cautious manner.

Provide contextual information that can help the users navigate or recover by themselves. Landmarks can be used to help the users disambiguate their location or orientation and proceed in the required path. Errorprevention (such as follow the wall on the left) or disambiguation (you should have the chairs on your right) instructions can be used when problematic areas are identified.

\subsection{Landmarks, POIs and Obstacles}

Users found valuable to know more about the environment, including POIs (e.g., water fountains or restrooms), obstacles to avoid (e.g, chairs, tables or columns) and landmarks that help them orient themselves and confirm their location (e.g., floor changes). As expected, the value of semantic information depended on the primary navigation aid: for instance, guide-dog users do not require information about obstacles $[14,18]$ and do not easily detect floor changes unless there is a clear difference (e.g., carpet to tile).

On the other hand, alerting users about the environment may create the expectation that the system will alert them about every element of interest in the route. However, this is not always the case, mainly due to changing conditions or 
even design decisions. The first includes doors that may be open when the user is asked to turn at the end of the corridor (leading the user to enter an office), or objects that are out of their place or were later added to the environment. Design decisions include areas with several POIs or obstacles where the system needs to prioritize instructions in order to avoid overloading the user.

Allow for personalization depending on the primary navigation aid and user needs/preferences. Allowing for different navigation modes (e.g., for guide-dog or white-cane users) can provide more adequate feedback to participants. However, it is important to consider that users rarely modify defaults and having too many settings may have a negative impact. Moreover, preferences and needs often change with knowledge, which is dynamic in the case of navigation for PVI. Possible approaches include modeling user behavior (e.g., [13]), which may allow for automatic adaptation of instructions based on the user and context.

\section{CONCLUSIONS}

We aimed to better understand how users behave in varying situational contexts while navigating with a smartphonebased turn-by-turn navigation app. We found that users' behaviors and coping mechanisms (e.g., anticipating a turn due to preventive behavior after a late turn) may lead to problematic situations, such as deviating from the intended path. In combination with particular scenarios, these behaviors can lead to errors and longer recovery times, even when the system behaves as expected. In order to inform further interface design and development that can accommodate the behavior of users, our study emphasized specific lessons learned and takeaways. The conclusions can be used by interface designers in order to provide more effective realtime feedback. The large deployment area and hours of data collected provided insights across a variety of users and operation modes. Although we used a specific navigational aid interface, the implications regarding user behavior can be applied to a broad set of navigation systems that rely on turn-by-turn instructions to guide PVI.

\section{ACKNOWLEDGEMENTS}

We thank the participants and the support of Shimizu Corporation, JST CREST (JPMJCR14E1) and NSF (1637927).

\section{REFERENCES}

[1] A. Abdolrahmani, W. Easley, M. Williams, S. Branham, and A. Hurst. Embracing errors: Examining how context of use impacts blind individuals' acceptance of navigation aid errors. In Proceedings of CHI, 2017.

[2] D. Ahmetovic, M. Murata, C. Gleason, E. Brady, H. Takagi, K. Kitani, and C. Asakawa. Achieving practical and accurate indoor navigation for people with visual impairments. Web for All, 2017.

[3] J. M. Coughlan and A. L. Yuille. Manhattan world: Compass direction from a single image by bayesian inference. In Proceedings of ICCV, 1999.

[4] J. Ducasse, A. M. Brock, and C. Jouffrais. Accessible interactive maps for visually impaired users. In Mobility of Visually Impaired People. 2018.
[5] N. Fallah, I. Apostolopoulos, K. Bekris, and E. Folmer. Indoor human navigation systems: A survey. Interacting with Computers, 2013.

[6] A. Fiannaca, I. Apostolopoulous, and E. Folmer. Headlock: a wearable navigation aid that helps blind cane users traverse large open spaces. In Proceedings of ASSETS. ACM, 2014.

[7] V. Filipe, F. Fernandes, H. Fernandes, A. Sousa, H. Paredes, and J. Barroso. Blind navigation support system based on microsoft kinect. Procedia Computer Science, 2012.

[8] J. Guerreiro, D. Ahmetovic, K. M. Kitani, and C. Asakawa. Virtual navigation for blind people: Building sequential representations of the real-world. In Proceedings of ASSETS. ACM, 2017.

[9] J.-E. Kim, M. Bessho, S. Kobayashi, N. Koshizuka, and K. Sakamura. Navigating visually impaired travelers in a large train station using smartphone and bluetooth low energy. In Proceedings of $A C M$ Symposium on Applied Computing. ACM, 2016.

[10] A. J. Kolarik, S. Cirstea, S. Pardhan, and B. C. Moore. A summary of research investigating echolocation abilities of blind and sighted humans. Hearing research, 2014.

[11] B. Li, J. P. Munoz, X. Rong, J. Xiao, Y. Tian, and A. Arditi. Isana: wearable context-aware indoor assistive navigation with obstacle avoidance for the blind. In Proceedings of ECCV. Springer, 2016.

[12] H. Nicolau, J. Jorge, and T. Guerreiro. Blobby: how to guide a blind person. In Proceedings of CHI EA. ACM, 2009.

[13] E. Ohn-Bar, J. Guerreiro, D. Ahmetovic, K. M. Kitani, and C. Asakawa. Modeling expertise in assistive navigation interfaces for blind people. In Proceedings of IUI, 2018.

[14] J. E. Pérez, M. Arrue, M. Kobayashi, H. Takagi, and C. Asakawa. Assessment of semantic taxonomies for blind indoor navigation based on a shopping center use case. In Proceedings of Web for All (W4A), 2017.

[15] D. Sato, U. Oh, K. Naito, H. Takagi, K. M. Kitani, and C. Asakawa. Navcog3: An evaluation of a smartphone-based blind indoor navigation assistant with semantic features in a large-scale environment. In Proceedings of ASSETS, 2017.

[16] M. K. Scheuerman, W. Easley, A. Abdolrahmani, A. Hurst, and S. Branham. Learning the language: The importance of studying written directions in designing navigational technologies for the blind. In Proceedings of CHI EA, 2017.

[17] W. R. Wiener, R. L. Welsh, and B. B. Blasch. Foundations of orientation and mobility. 2010.

[18] M. A. Williams, A. Hurst, and S. K. Kane. Pray before you step out: describing personal and situational blind navigation behaviors. In Proceedings of ASSETS, 2013.

[19] L. Zeng, M. Simros, and G. Weber. Camera-based mobile electronic travel aids support for cognitive mapping of unknown spaces. In Proceedings of MobileHCI, 2017. 